\title{
Editorial
}

\author{
Elmar Mittler*
}

\section{BIBLIOTHEK - Forschung und Praxis und Open Access}

https://doi.org/10.1515/bfp-2020-2095

„Jetzt auch im INTERNET“ war die Information auf der Titelseite von Heft 1 des 20. Jahrgangs 1996 von BFP. Damit war die Zeitschrift auf einem Göttinger Server schon Open Access, bevor dieser Begriff weltweit seinen Siegeszug antrat. Als der Verlag auch die elektronische Publikation mit einer Embargozeit von einem Jahr bis zur OA-Bereitstellung übernahm, wurden die Manuskripte (mit einer kleinen Unterbrechung nach dem Wegfall von b2i) nach Annahme als Preprints Open Access gestellt. ${ }^{1}$ Der Verlag bereitet in Zusammenarbeit mit den Herausgebern die Umstellung der Zeitschrift ab 2021 auf Open Access nach dem Modell „Subscribe to Open“ (S2O) vor. Damit stehen in Zukunft alle Beiträge sofort in der qualitativ hochwertigen und zitierfähigen Verlagsversion Open Access zur Verfügung. Der Nationale Open-Access-Kontaktpunkt unterstützt diese Transformation nach dem S2O-Modell. Er hat auf seiner Webseite eine Information veröffentlicht, die hier im Wortlaut wiedergegeben wird. ${ }^{2}$

Wir hoffen sehr, dass die geplante Umstellung gelingt, und bitten unsere Abonnenten und Leser dabei um Unterstützung.

\section{Subscribe to Open „BIBLIOTHEK- Forschung und Praxis“}

Laufzeit: ab 2021

Organisatoren: De Gruyter Verlag, Editorial Board BIBLIOTHEK-Forschung und Praxis, Abonnenten der Zeitschrift Verlag: De Gruyter

\section{Hintergrund}

Das Subscribe to Open-Modell wurde von Annual Reviews als Ansatz für die Open-Access-Transformation gut einge-

1 https://edoc.hu-berlin.de/handle/18452/19065

2 Englisch: https://oa2020-de.org/en/pages/S2O_BFP

*Kontaktperson: Prof. Dr. Dr. h.c. Elmar Mittler, emittle@gwdg.de führter Subskriptionszeitschriften entwickelt und nutzt bestehende Kunden- und Abonnentenbeziehungen sowie Rechnungsworkflows nach. Einrichtungen, die die Inhalte der jeweiligen Zeitschriften kennen und schätzen, abonnieren diese wie gewohnt weiter, während die Inhalte Open Access erscheinen. Dadurch fallen für das Publizieren keine APCs oder sonstigen zusätzlichen Kosten an. Solange die vorhandenen Abonnements erhalten und verlängert werden, erscheint die Zeitschrift Open Access unter einer Creative-Commons-Lizenz. Fällt die Anzahl der Abos unter einen vorher festgelegten Wert, wird die „Paywall“ wieder aktiv, d.h. nur Abonnenten haben Zugriff.

Mit diesem Modell lassen sich mit wenig Aufwand und ohne Mehrkosten komplette Zeitschrifteninhalte in den Open Access transfomieren. Sein Potential liegt daher vor allem in der Aktivierung der subskribierenden Einrichtungen, durch das Beibehalten eines Abonnements den freien Zugang zu den Inhalten für alle zu garantieren.

Bisher haben neben Annual Reviews mit dem Journal Annual Review of Cancer Biology vor allem der Verlag Berghahn mit dem Berghahn Open Anthro Projekt und EDP Science mit seinem Journal Mathematical Modelling in Natural Phenomena das Modell umgesetzt. In Deutschland setzt der Nomos-Verlag mit der neu gegründeten OpenAccess-Zeitschrift Recht und Zugang auf dieses Modell und baut zur Finanzierung einen neuen Abonnenten-Stamm auf.

\section{Konditionen}

Die Abonnenten co-finanzieren durch ihr Abo die Open-Access-Publikation der Zeitschrift BIBLIOTHEKForschung und Praxis. Die Teilnahme erfolgt im Rahmen der jährlichen Aboerneuerung und als zusätzlichen Anreiz gibt es garantierte Preisstabilität für die Jahrgänge 2021 und 2022. Ansonsten ändert sich für die Bibliotheken nichts gegenüber ihrem normalen Abonnement.

\section{Vorteile}

Bei der Unterstützung von Subscribe to Open (S2O) finanzieren bestehende Bibliotheken weiterhin die von ihnen als relevant eingeordneten Zeitschriften, während neu hinzukommende Bibliotheken ein Modell abonnieren, das es 
allen Lesern und Autoren ermöglicht, von Open Access zu profitieren und das die Finanzierung eines nachhaltigen Open Access, insbesondere für kleinere Zeitschriften in den Sozial- und Geisteswissenschaften, leichter zugänglich macht. S2O bietet eine einfache und unkomplizierte Möglichkeit des Übergangs zu Open Access, basierend auf bibliothekarischer Kuratierung, vorhandenen Ressourcen und bewährten Prozessen zur Unterstützung von Zeitschriften.

\section{Mitmachen}

Das S20-Modell der Zeitschrift BIBLIOTHEK-Forschung und Praxis können Sie ganz einfach unterstützen, in dem Sie Ihr bestehendes Abonnement auf den gewohnten Wegen verlängern und für 2021 weiterführen.
Weiterführende Informationen finden Sie unter www. degruyter.com/subscribe-to-open. Bei Fragen steht Ihnen Dr. Christina Lembrecht (christina.lembrecht@degruyter. com) zur Verfügung.

\section{Prof. Dr. Dr. h.c. Elmar Mittler}

Niedersächsische Staats- und Universitätsbibliothek

Papendiek 14

D-37073 Göttingen

emittle@gwdg.de 